\title{
Prophylactic Effect of Lactobacillus pentosus strain S-PT84 on Candida Infection and Gastric Inflammation in a Murine Gastrointestinal Candidiasis Model
}

\author{
Toshihiro Maekawa ${ }^{1}$, Sanae A. Ishijima ${ }^{2}$, Masayuki Ida ${ }^{1}$, Takayuki Izumo ${ }^{1}$, Yoshiko Ono ${ }^{1}$, \\ Hiroshi Shibata ${ }^{1}$ and Shigeru Abe ${ }^{2}$
}

${ }^{1}$ Institute for Health Care Science, Suntory Wellness Limited

${ }^{2}$ Teikyo University Institute of Medical Mycology

\begin{abstract}
We previously showed a prophylactic effect of Lactobacillus pentosus strain S-PT84 against oral candidiasis in mice. In the present study, we evaluated the protective effect of S-PT84 against Candida infection of the gastrointestinal tract. As the first step, we used an in vitro assay to compare the inhibitory effects of several lactobacilli (S-PT84 and Lactobacillus pentosus type strain JCM1558', Lactobacillus gasseri type strain $\mathrm{JCM} 1131^{\top}$ and Lactobacillus casei type strain $\mathrm{JCM} 1134^{\top}$ ) on mycelial growth of Candida albicans. S-PT84 directly adhered to Candida cells and showed the strongest growth-inhibitory activity among the tested Lactobacillus strains. In the second experiment, we used an in vivo assay to evaluate the effect of S-PT84 ingestion on severity score of stomach lesion and gastric inflammation in a mouse model of gastrointestinal candidiasis. The severity scores were significantly improved by oral administration of S-PT84 $(6 \mathrm{mg} / 200 \mu \mathrm{L})$, consistent with decreased coverage of stomach lesions by patchy whitish plaques. The attenuation of stomach lesion severity by S-PT84 was more pronounced than that obtained with $L$. gasseri type strain $\mathrm{JCM} 1131^{\top}$, consistent with the results of the above in vitro study. Histological analysis also indicated that S-PT84 prevented the adhesion of $C$. albicans to the stomach surface and suppressed stomach inflammation caused by neutrophil infiltration. Furthermore, S-PT84 also suppressed the vascular permeability observed in Candidainfected stomach. These results suggest that oral administration of S-PT84 might be effective not only in inhibiting Candida infection but also in preventing gastric inflammation induced by Candida infection.

Key words : Candida albicans, infection, inflammation, Lactobacillus pentosus strain S-PT84, permeability
\end{abstract}

\section{Introduction}

Candida albicans is one of the opportunistic pathogens constituting the human flora and found as commensal eumycetes on skin, genitourinary, and gastrointestinal tracts ${ }^{1)}$. These microorganisms can be isolated from the oral cavities of $20 \%$ to $80 \%$ of healthy individuals ${ }^{2,3)}$, with $C$. albicans colonization of the tongue reported to increase with age $^{4)}$. Some factors, such as immunosuppression or use of broad-spectrum antimicrobial drugs, can induce the overgrowth of Candida, resulting in the development of candidiasis ${ }^{5)}$ in any of several tissues, including the gastrointestinal, respiratory, or genital tracts. For example, several investigators reported that $C$. albicans was isolated from gastric ulcer or ulcerative colitis, ${ }^{6,7)}$, and dyspeptic diseases were associated with the co-infection of $C$. albicans and Helicobacter pylori ${ }^{8)}$. These reports show that it is a necessary to prevent the infection of $C$. albicans in the gastrointestinal tract to maintain health.

Address for correspondence : Toshihiro Maekawa

Institute for Health Care Science, Suntory Wellness Limited, 8-1-1 Seikadai, Seika-cho, Soraku-gun, Kyoto 619-0284, Japan

Received : 2, May 2016, Accepted : 21, September 2016

E-mail : Toshihiro_Maekawa@suntory.co.jp 
It is well known that interactions between $C$. albicans and commensal bacteria (such as Streptococcus, Staphylococcus, and Lactobacillus) can influence the health of the host ${ }^{9-14)}$. For example, Streptococcus has been reported to inhibit the mycelial growth of $C$. albicans in the oral cavity; this bacterium could be useful for prevention of oral candidiasis. Other works have shown that heat-killed Enterococcus faecalis directly adheres to $C$. albicans, and that this binding leads to inhibition of attachment of $C$. albicans cells to the substratum. Indeed, oral administration of $E$. faecalis has been shown to have a therapeutic effect in a mouse model of oral candidiasis $^{15)}$.

Lactobacillus pentosus strain S-PT84 was originally isolated from Kyoto pickles, and we reported several physiological functions of this strain both in vitro and in vivo. S-PT84 induces IL-12 production from peritoneal macrophages in vitro, and enhances splenic natural killer (NK) cell activity in $v^{\prime}{ }^{16)}$. Izumo et al. reported that intranasal administration of S-PT84 in mice augmented NK activity in the lung, increased alpha-interferon production in bronchoalveolar lavage fluids, and protected against influenza virus infection ${ }^{17)}$. Moreover, the ingestion of S-PT84 increased the level of Salmonella-specific antibodies in the small intestine and provided a reduction in the number of Salmonella typhimurium cells in feces in Salmonella-infected mice ${ }^{18)}$. Hayama et al. reported that S-PT84 inhibited mycelial growth of $C$. albicans strain TIMM1768 in vitro, protecting the host from $C$. albicans infection and reducing lesional scores on tongues in a murine oral candidiasis model $^{19)}$. Considered together, these results suggest that S-PT84 has the potential to attenuate the severity of gastrointestinal tract lesions associated with Candida infection.

Recently, a novel murine gastrointestinal candidiasis model using N-acetylglucosamine (GlcNAc) was developed; this model employs a new symptom scoring system that assesses the extent of the patchy whitish plaques on mucosal epithelium in the gastric region ${ }^{20)}$. In this model, histological analysis of Candida-infected stomach reveals the presence of both thick Candida mycelia invading the mucosal epithelial tissue and infiltrating inflammatory cells. Therefore, we hypothesized that this murine model would be suitable for assessment of the symptoms of gastric lesions and for histological analysis of inflammation associated with gastrointestinal candidiasis.

In the present study, we investigated the ability of S-PT84 to prevent gastrointestinal candidiasis. Specifically, we tested the amelioration of the severity score and inflammatory state in the stomach by oral administration of S-PT84 in a murine model of gastrointestinal candidiasis. In addition, we compared the protective effect of SPT84 against Candida infection to that obtained with other Lactobacillus type strains.

\section{Materials and Methods}

\section{Preparation of Candida albicans and lactobacilli}

C. albicans strain TIMM1768 was isolated clinically from the blood of a candidiasis patient and stored at Teikyo University Institute of Medical Mycology. This strain, which has been shown to induce oral candidiasis in a murine model, has been used in several previous animal experiments ${ }^{19-21)}$. Cultures were stored at $-80^{\circ} \mathrm{C}$ in Sabouraud dextrose broth (Becton Dickinson, MD, USA) containing $0.5 \%$ yeast extract (Becton Dickinson) and $10 \%$ glycerol ( $\mathrm{v} / \mathrm{v}$, final concentration) until use. TIMM1768 was cultured on a Sabouraud dextrose agar plate for $18 \mathrm{~h}$ at $37^{\circ} \mathrm{C}$, and the cells were harvested with a micro-spatula and suspended in RPMI1640 (Sigma Chemical Co., St. Louis, MO, USA) -based growth medium. This growth medium consisted of RPMI1640 diluted 1:3 and supplemented with $0.8 \%$ heat-inactivated fetal calf serum, $20 \mathrm{mM}$ HEPES buffer (pH7.2), $2 \mathrm{mM}$ urea, $10 \mathrm{mg} / \mathrm{ml}$ D-glucose, and antibiotics (60 $\mu \mathrm{g} / \mathrm{ml}$ of benzyl penicillin potassium [Wako] and kanamycin sulfate [Wako]). The cultured $C$. albicans cells were used for in vitro germ tube formation, for a mycelial growth experiment, and for in vivo oral infection with Candida.

S-PT84 was isolated from Kyoto pickles "SHIBAZUKE"16) and was cultivated in a medium containing glucose and yeast extract (Aromild ${ }^{\mathrm{TM}}$, $\mathrm{SK}$ yeast extract $\mathrm{Hi}-\mathrm{K}$ ) at $37^{\circ} \mathrm{C}$ for $24 \mathrm{~h}$. Cultured bacteria were collected by centrifugation and heat-killed at $90^{\circ} \mathrm{C}$ for $30 \mathrm{~min}$. Heat-killed S-PT84 was lyophilized pending formulation for dosing in the study.

We prepared three other Lactobacillus type strains for comparison: JCM1558 ${ }^{\top}$ (Lactobacillus pentosus type strain), JCM1131 ${ }^{\top}$ (Lactobacillus gasseri type strain), and JCM1134 ${ }^{\top}$ (Lactobacillus casei type strain). All type strains were obtained from the Japan Collection of Microorganisms 
(JCM), Riken, Japan. These organisms were cultured as for S-PT84. Cultured bacteria were then collected by centrifugation, washed twice with sterile saline, washed once with distilled water, and heat-killed at $95^{\circ} \mathrm{C}$ for $5 \mathrm{~min}$. Heat-killed lactobacilli were lyophilized for use in this study.

\section{In vitro assay of crystal violet staining}

A suspension (in diluted RPMl1640-based medium) of $C$. albicans cells (at $5 \times 10^{2}$ colony forming units [CFU] per well for mycelial growth), with or without heat-killed bacteria (S-PT84, $L$. pentosus JCM1558', L. gasseri JCM1131' ${ }^{\top}$, or $L$. casei $\mathrm{JCM} 1134^{\top}$ ), was distributed to 96 -well plates at $200 \mu \mathrm{l} /$ well. After incubation at $37^{\circ} \mathrm{C}$ in $5 \% \mathrm{CO}_{2}$ in air for $20 \mathrm{~h}$, the medium in the wells was discarded, and the $C$. albicans cells were sterilized and fixed by immersion of the plate in $70 \%$ ethanol for $2 \mathrm{~min}$. The wells were then rinsed twice with distilled water. The mycelia attached to the bottom of the wells were stained by exposure to crystal violet (CV; $0.02 \%$ in phosphate-buffered saline, 100 $\mu \mathrm{l} /$ well) for $20 \mathrm{~min}$. Wells were washed 3 times with water, once with $0.0625 \%$ sodium dodecyl sulfate (SDS) and twice more with water. After drying of the microtiter plates, CV was extracted from the mycelia by addition of isopropanol-0.04 M $\mathrm{HCl}(150 \mu \mathrm{l} /$ well) and $0.25 \%$ SDS $(50 \mu \mathrm{l} /$ well) to each well followed by mixing on a plate mixer for 2 min. The absorbance at $620 \mathrm{~nm}$ of quintuplicate samples was measured photometrically.

\section{Yeast viability assay using fluorescence microscopy}

The effect of S-PT84 on C. albicans viability was evaluated using a live or dead yeast viability assay that employs a two-color fluorescent probe (FUN1; F-7030; Molecular Probes, Eugene, OR, USA). C. albicans cells were cultured (using sufficient volumes of culture medium) on poly-Llysine-coated glass cover slips for $1 \mathrm{~h}$ at $27^{\circ} \mathrm{C}$ (yeast form) or $37^{\circ} \mathrm{C}$ (hyphal form); heat-killed SPT84 cells were added to each cover slip and culturing was continued at the respective temperature for another $3 \mathrm{~h}$ in a $5 \% \mathrm{CO}_{2}$ incubator. Cover slips were centrifuged at 3,000 rpm for $3 \mathrm{~min}$ and washed once with $\mathrm{GH}$ solution ( $2 \%$ glucose in 10 mM HEPES buffer, $\mathrm{pH} 7.2$ ); the $\mathrm{GH}$ solution was then replaced with $\mathrm{GH}$ solution containing $20 \mu \mathrm{M}$ FUN1. After incubation for $30 \mathrm{~min}$ at room temperature, cells were observed with a fluorescent microscope (BH50, Olympus, Japan) equipped with an assortment of filters, including: WBV (wide range of blue-violet excitation), WG (wide range of green excitation), and NB (narrow range of UV excitation). Staining of FUN1 was observed using the NB filter. All images were recorded as digital data with a DC200 camera (Leica, Germany) and were analyzed using the IM50 program.

\section{Murine model of gastrointestinal candidiasis}

We performed animal experiments in accordance with procedures consistent with Teikyo University's "Guidelines for the Care and Use of Animals." The murine gastrointestinal candidiasis model has been described previously ${ }^{20)}$. All experiments involved six-week-old female ICR mice (Charles River Japan, Inc., Yokohama). Mice were maintained in cages housing two to four individuals and were provided with ad libitum access to a commercial diet (Labo MR Stock, Norsan Corporation Life-Tech Department, Kanagawa, Japan) and tap water. During the experimental period, photoperiods were adjusted to a daily 12 h-12 h light-dark cycle with environmental temperature maintained at $26 \pm 0.5^{\circ} \mathrm{C}$. The experimental procedure used to establish gastrointestinal candidiasis in mice was as follows. To induce an immunosuppressed condition, we injected mice with $100 \mathrm{mg} / \mathrm{kg}$ of prednisolone (Kyoritsu Seiyaku Co., Japan) subcutaneously 22 $\mathrm{h}$ to $24 \mathrm{~h}$ before intragastric inoculation with $C$. albicans. Mice were given drinking water with 4.2 $\mathrm{mg} / \mathrm{ml}$ tetracycline hydrochloride (CTC, SC Environmental Science Co. Ltd., Japan) and $50 \mathrm{mM}$ $\mathrm{N}$-acetylglucosamine (GlcNAc) starting from $48 \mathrm{~h}$ before the inoculation with $C$. albicans and continuing throughout the experimental period. After a 3-hour fast, mice were infected by intragastric inoculation with $2 \times 10^{8} \mathrm{CFU} /$ mouse of $C$. albicans TIMM1768 cells. Cells were administered as $0.2 \mathrm{ml} /$ mouse of a suspension in modified RPMI1640 medium, using a syringe with a roundheaded needle (39-mm length; Fuchigami, Ltd., Kyoto). Needle resistance at the stomach wall was used as an indication that $C$. albicans was properly injected. At $1.5 \mathrm{~h}$ after gastric inoculation with Candida, mice were sedated by injection of chlorpromazine chloride (3-(2-chloro-10H-phenothiazin-10-yl)-N, N-dimethylpropylamine monohydrochloride) at $14.4 \mathrm{mg} / \mathrm{kg}$ in the femoral muscle.

Administration of lactobacilli in murine model of gastrointestinal candidiasis

Mice were separated into four groups as follows: normal group (Candida non-infected), control group (Candida infected), S-PT84 group (Candida infected + S-PT84), and L. gasseri $\mathrm{JCM} 131^{\top}$ group (Candida infected + L. gasseri 
JCM1131 ${ }^{\top}$ ). Lyophilized S-PT84 and L. gasseri $\mathrm{JCM} 1131^{\top}$ each were formulated at $30 \mathrm{mg} / \mathrm{ml}$ in saline and administered at $0.2 \mathrm{ml} /$ mouse by intragastric gavage using a round-headed needle; mice in the normal and control groups were administered an equivalent volume of saline. Intragastric gavages were performed $3 \mathrm{~h}$ after Candida inoculation.

Assessment of severity score and fungal burden in murine model of gastrointestinal candidiasis

At $48 \mathrm{~h}$ after Candida inoculation, mice were sacrificed by cervical dislocation, and their stomachs were removed. The stomachs were then cut open along the longitudinal axis. Stomach contents were removed with tweezers and the stomachs were washed twice with $5 \mathrm{ml}$ of sterile saline. We scored the severity of stomach lesions based on the degree of coverage with patchy whitish plaques coating the total inner stomach surface using the following rating system ${ }^{20)}: 0$, normal; 1 , patchy whitish plaques covering less than $20 \%$; 2, patchy whitish plaques covering $20 \%$ or more, but less than $50 \%$; 3 , patchy whitish plaques covering $50 \%$ or more, but less than $90 \%$, and resembling a squamous layer; 4, patchy whitish plaques covering $90 \%$ or more of the stomach and on the squamous layer; 5, thick, patchy whitish plaques covering $90 \%$ or more of the stomach and on the squamous layer. Upon completion of scoring, the CFU of Candida cells in the stomachs were quantified as follows. The stomach weights were recorded, and each organ was homogenized in $3 \mathrm{ml}$ of sterile saline. The resulting homogenates were subjected to serial 20 -fold and 100-fold dilutions in sterile saline, and 50 $\mu$ I of each dilution were plated to Candida GS agar (selection medium for Candida; Eiken Chemical Co. Ltd., Japan). Plates were incubated for $20 \mathrm{~h}$ at $37^{\circ} \mathrm{C}$, and colony numbers were determined. Candida cell densities were calculated as CFU per gram of organ.

\section{Assessment of vascular permeability by tail vein injection of Evans blue}

We evaluated vascular permeability using the murine gastrointestinal candidiasis model as described previously ${ }^{20)}$. Mice were separated into three groups as follows: normal group $(n=4)$, control group $(n=4)$, and S-PT84 group $(n=4)$. SPT84 was administered once at $3 \mathrm{~h}$ after Candida infection. At $48 \mathrm{~h}$ after Candida infection, mice were injected via the tail vein (at $10 \mathrm{ml} / \mathrm{kg}$ ) with Evans blue ( $0.5 \%$ in saline). Animals were sacri- ficed by cervical dislocation 20 min after tail vein injection, and the stomachs were collected. The stomachs were slit open and washed with cold saline. The frozen stomachs were ground using a Polytron PT1200, and acetone was added to the resulting ground tissue at $2 \mathrm{ml} / \mathrm{stomach}$. These suspensions were vortexed for $10 \mathrm{~min}$ and then centrifuged at $3500 \mathrm{rpm}$ for $20 \mathrm{~min}$. The resulting supernatant was diluted four-fold in acetone, and absorbance was measured at a wavelength of 600 $\mathrm{nm}$.

Histology

For histological evaluation, the stomachs were resected at the base, fixed with phosphatebuffered $4 \%$ paraformaldehyde solution $(\mathrm{pH} 7.4)$ at $4^{\circ} \mathrm{C}$, dehydrated using a graded ethanol series, and embedded in paraffin in accordance with standard procedures. Specimens were sectioned at $8-\mu \mathrm{m}$ thicknesses along the longitudinal centerline and placed on slides. Sections then were deparaffinized in xylene and rehydrated using a graded ethanol series; fungal cell walls were stained with the Periodic Acid Schiff (PAS) reaction. Sections were counter-stained with hematoxylin-eosin (HE).

Anti-neutrophil antibody staining was performed as follows. Sections were cut at 3- to $5-\mu \mathrm{m}$ thicknesses along the longitudinal centerline and placed on slides, then deparaffinized in xylene and rehydrated using a graded ethanol series. Slides were rinsed with deionized water and Trisbuffer saline (TBS, pH 7.6). Antigen retrieval was performed in a pressure cooker by incubating for $10 \mathrm{~min}$ at $120^{\circ} \mathrm{C}$ in $0.01 \mathrm{M}$ citrate-buffered solution $(\mathrm{pH}$ 7.2). Slides were rinsed three times with TBS, treated with $1 \%$ hydrogen peroxide in methanol, and rinsed another three times with TBS. The slides were incubated with avidin solution (Biotin Blocking System, catalog no. X0590; Agilent Technologies, Santa Clara, CA, USA) for $10 \mathrm{~min}$, rinsed three times with TBS, incubated with biotin solution (Biotin Blocking System; Agilent Technologies) for $10 \mathrm{~min}$, and rinsed another three times with TBS. After blocking of nonspecific binding with skim milk, the slides were incubated for $1 \mathrm{~h}$ at $4^{\circ} \mathrm{C}$ with rabbit anti-neutrophil elastase polyclonal antibody (1:100; catalog no. bs-6982R; Bioss Inc., Woburn, MA, USA), rinsed three times with TBS, and incubated for $30 \mathrm{~min}$ at room temperature with biotinylated secondary antibody (polyclonal goat anti-rabbit immunoglobulins, biotinylated; catalog no. E0432; Agilent Technologies). The slides were 
then rinsed three times with TBS and incubated for 30 min at room temperature with $A B C$ reagent (Ultra-Sensitive ABC Peroxidase staining Kits; catalog no. 32050; Thermo Fisher Scientific, Waltham, MA, USA). After three additional washes with TBS, the slides were incubated for 3 min with the enzymatic substrate (DAB substrate kit; catalog no. 34065; Thermo Fisher Scientific); reactions were quenched by immersion in deionized water. Sections were counter-stained with Mayer's hematoxylin.

\section{Statistical analysis}

The significance of differences between the normal and control groups was assessed using the unpaired Student's t-test (two-tailed); significance of differences between the control and experimental groups was determined using the unpaired Student's t-test or Tukey's HSD test (two-tailed). The correlation between the incorporation of Evans blue and score of stomach lesions was determined using the Pearson's correlation coefficient test.

For all tests, $\mathrm{p}$ values less than 0.05 were considered statistically significant. Statistical analyses were performed with SPSS statistical software (IBM SPSS Statistics, version 23).

\section{Results}

\section{S-PT84 inhibits mycelial growth of $C$. albicans}

We used S-PT84 and several Lactobacillus type strains ( $L$. pentosus JCM1558 ${ }^{\top}, \quad L$. gasseri $\mathrm{JCM} 1131^{\top}$, and $L$. casei $\mathrm{JCM} 1134^{\top}$ ). All lactobacilli inhibited mycelial growth of $C$. albicans in a dosedependent manner. S-PT84 exhibited the strongest inhibitory activity among these four lactobacilli strains, whereas L. gasseri JCM1131 ${ }^{\top}$ was the weakest (Fig. 1).

S-PT84 selectively inhibits the hyphal form of $C$. albicans

To determine the viability of $C$. albicans by evaluating the metabolic activity, we stained $C$. albicans with FUN1 (FUN1 assay). In this system, FUN1 is accumulated in the vacuoles in the cytoplasm and converts into red fluorescent compounds by metabolism of living cells. On the other hand, a broad green accumulate is observed in a dead cell. For both hyphal (Fig. 2A) and yeast (Fig. 2B) forms of C. albicans, red particles were observed, i. e., C. albicans cells were metabolically active. Upon incubation with S-PT84, both hyphal and yeast forms of $C$. albicans

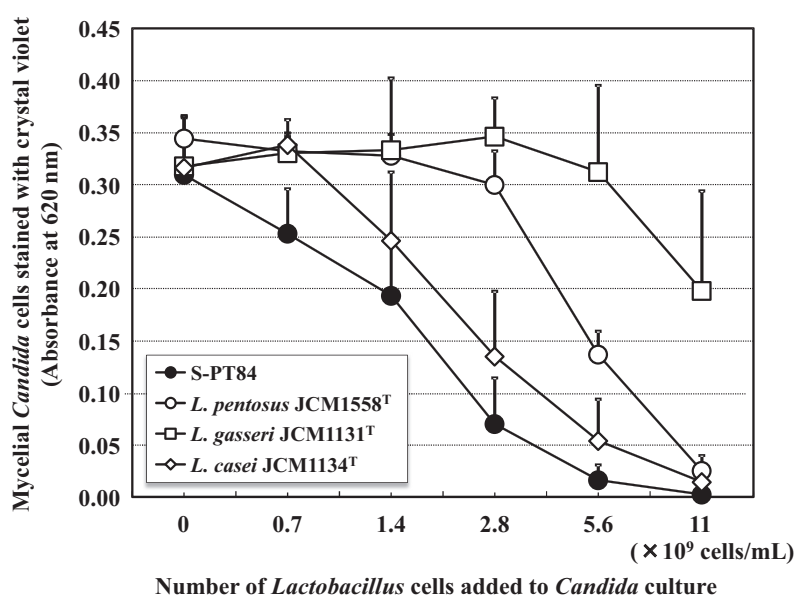

Fig. 1 Inhibitory effect of lactobacilli on mycelial growth of $C$. albicans strain TIMM1768.

C. albicans was cultured with different doses of SPT84, L. pentosus JCM1558 ${ }^{\top}$ (L. pentosus type strain), L. gasseri $J C M 1131^{\top}$ (L. gasseri type strain), or $L$. casei JCM1134 $4^{\top}$ (L. casei type strain) for $20 \mathrm{~h}$ at $37^{\circ} \mathrm{C}$ in a $5 \% \mathrm{CO}_{2}$ environment. The dosage of S-PT84 at 0.75 $\times 10^{9}$ cells $/ \mathrm{ml}$ was equivalent to that of $1 \mathrm{mg} / \mathrm{ml}$. The dosages of $L$. pentosus JCM $1558^{\top}$ at $1.49 \times 10^{9} \mathrm{cells} / \mathrm{ml}$, L. gasseri JCM $1131^{\top}$ at $1.53 \times 10^{9} \mathrm{cells} / \mathrm{ml}$ and $L$. casei JCM1134 at $1.36 \times 10^{9}$ cells $/ \mathrm{ml}$ were equivalent to those of $1 \mathrm{mg} / \mathrm{ml}$. C. albicans cells were stained with crystal violet. The optical absorbance at $620 \mathrm{~nm}$ was detected. Data are shown as means \pm SD $(n=3-8)$.

cells were surrounded by small green bacilli (SPT84) (Fig. 2C, 2D, 2E, 2F). Though yeast-form cells accumulated red particles in $C$. albicans cells (Fig. 2D, 2F), hyphal-form cells displayed few or no red particles (Fig. 2C, 2E). These results indicated that S-PT84 adhered to $C$. albicans cells (both hyphal and yeast forms), but S-PT84 appeared to selectively suppress the metabolic activity of the hyphal form of $C$. albicans.

S-PT84 ameliorates the severity scores of stomach lesions

We investigated the protective effect of S-PT84 and $L$. gasseri $\mathrm{JCM} 1131^{\top}$ against worsening of symptoms and Candida colonization in the stomach. Fig. 3A shows the severity scores of stomach lesions. Administration of S-PT84 (6 $\mathrm{mg} / 200 \mu \mathrm{l}$ ) ameliorated the severity score of stomach lesions compared to that of the control group (2.33 \pm 0.52 vs. $4.40 \pm 0.55 ; p<0.001$ ). Administration of $L$. gasseri $\mathrm{JCM} 1131^{\top}$ also improved the severity score $(3.33 \pm 0.52, p<0.05)$ compared with that of the control group; however, its effect was significantly weaker than that of SPT84 $(p<0.05)$. The viable cell number in the 

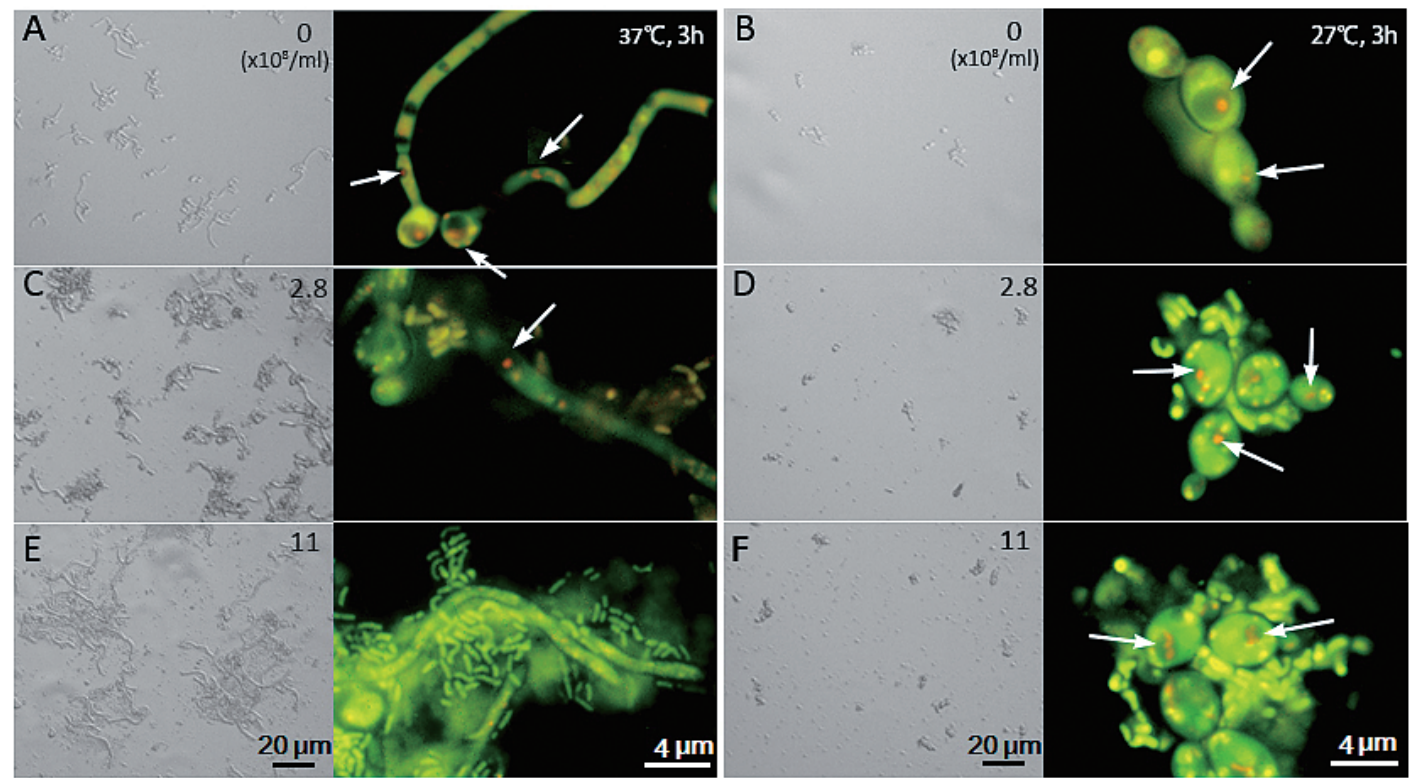

Fig. 2 Representative images of vital staining of $C$. albicans by FUN1.

C. albicans was cultured with or without S-PT84 at $27{ }^{\circ} \mathrm{C}$ or $37^{\circ} \mathrm{C}$ for $3 \mathrm{~h}$ and stained with FUN1. A; Hyphal forms of $C$. albicans cultured without S-PT84. B; Yeast forms of $C$. albicans cultured without S-PT84. C, E; Hyphal forms of $C$. albicans cultured with S-PT84 $\left(2.8 \times 10^{8}\right.$ or $\left.11 \times 10^{8} \mathrm{cells} / \mathrm{ml}\right)$. D, F; Yeast forms of $C$. albicans cultured with S-PT84 $\left(2.8 \times 10^{8}\right.$ or $11 \times 10^{8}$ cells $\left./ \mathrm{ml}\right)$. White arrows indicate the accumulated red crystals of FUN1. Scale bars are as indicated.

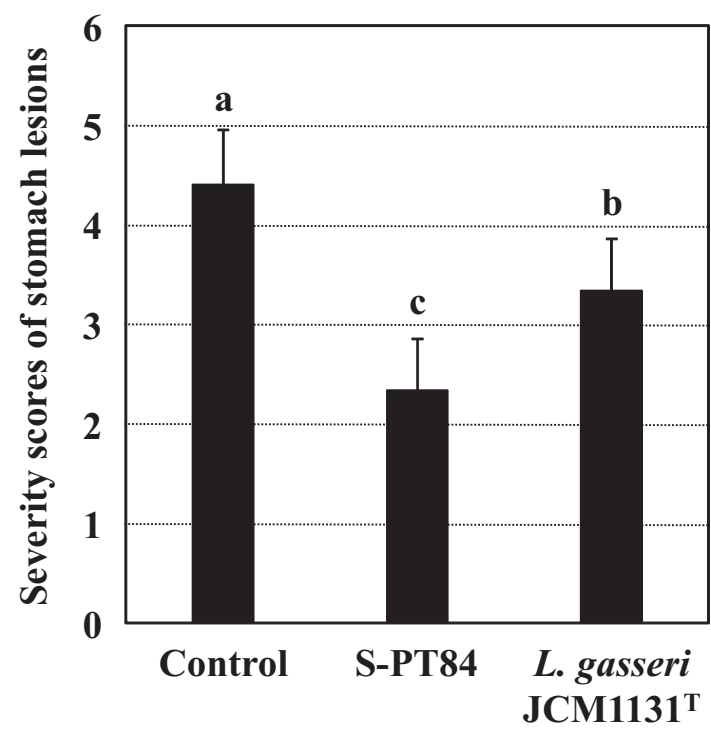

(A)

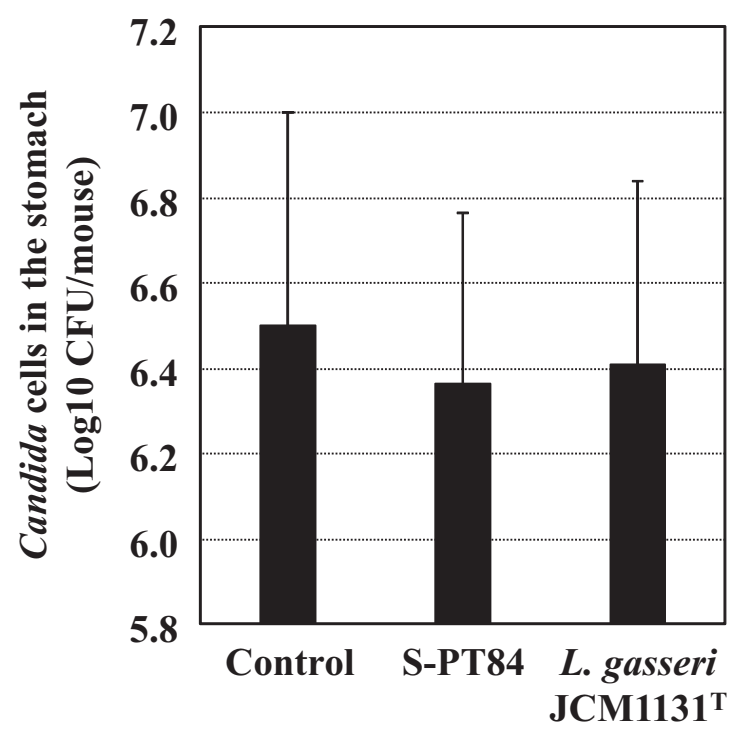

(B)

Fig. 3 Effect of S-PT84 and $\mathrm{JCM} 1131^{\top}$ on the severity score and the viable cell number of $C$. albicans strain TIMM1768.

Mice were allocated to three groups: control group (infected; $n=5$ ), S-PT84 group (infected $+\mathrm{S}-\mathrm{PT} 84 ; \mathrm{n}=6$ ), and L. gasseri $\mathrm{JCM} 1131^{\top}$ group (infected + L. gasseri $\mathrm{JCM} 1131^{\top} ; \mathrm{n}=6$ ). S-PT84 and L. gasseri $\mathrm{JCM} 1131^{\top}$ were administered as described in Methods. Animals were sacrificed and their stomachs were removed at $48 \mathrm{~h}$ after Candida infection, as described in Methods. Severity scores of stomach lesions (indicative of the degree of formation of patchy whitish plaques; $A$ ) and densities of $C$. albicans viable cell in homogenized stomachs (B) were determined as described in Methods. Data are presented as mean $\pm S D(n=5$ or 6$)$. Labeled means without a common letter are significantly different by Tukey's HSD test $(P<0.05)$. 


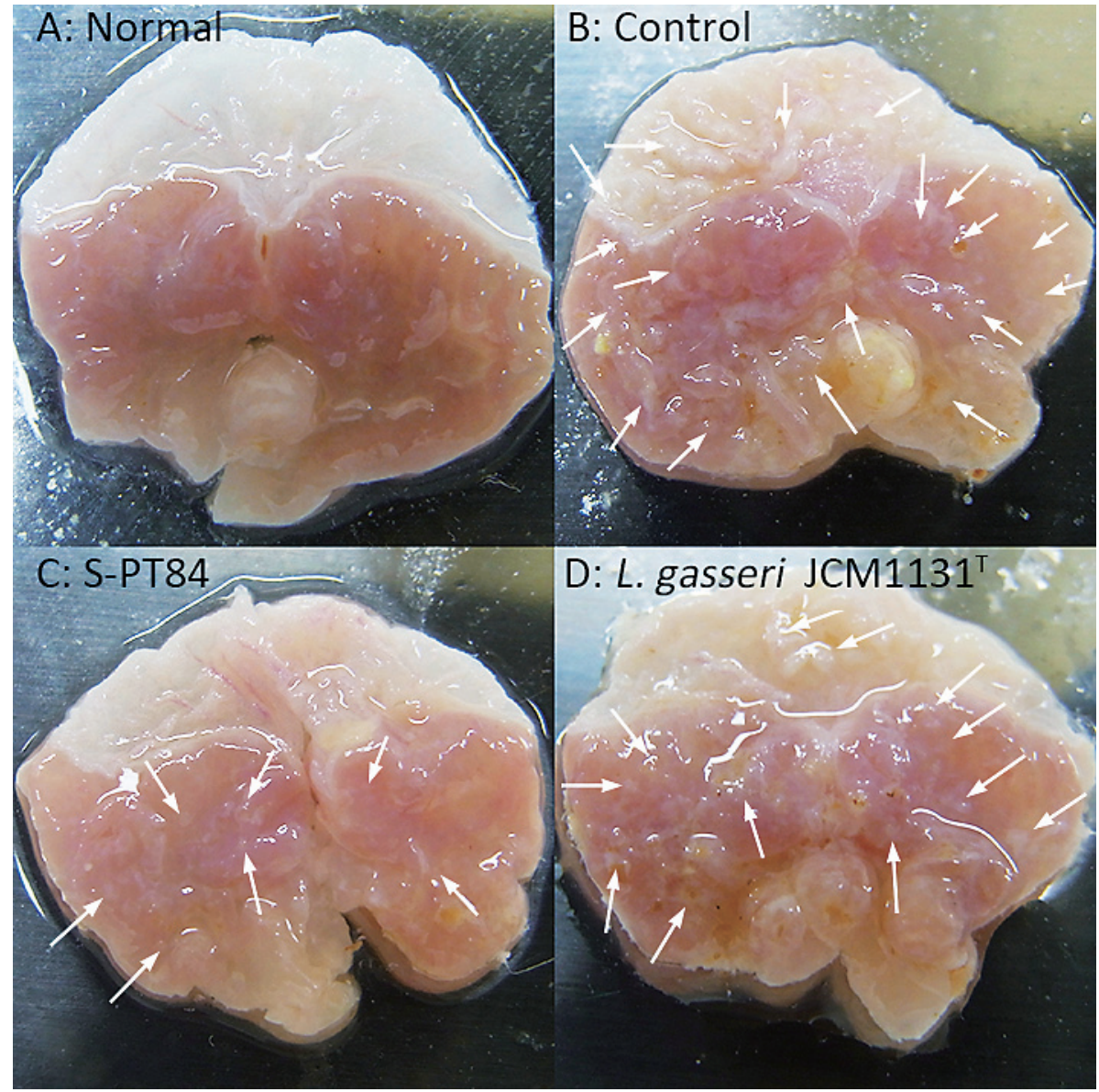

Fig. 4 Representative inner surface images of stomachs $48 \mathrm{~h}$ after infection with C. albicans strain TIMM1768.

(A) Normal group (non-infected), (B) Control group (infected), (C) S-PT84 group (infected + S-PT84), (D) L. gasseri JCM $1131^{\top}$ group (infected + L. gasseri $J C M 1131^{\top}$ ). Images are representative for each semi-quantitative score. White arrows indicate the presence of patchy whitish plaques.

stomach was not significantly different among the groups (Fig. 3B).

Fig. 4 shows typical features of the tissues. Coverage of the patchy whitish plaques (white arrow) on the inner surface was observed in a broad area in the control group. In contrast, the degree of coverage of the patchy whitish plaques in both S-PT84 group and L. gasseri JCM1131 ${ }^{\top}$ groups was evidently decreased compared to that in the control group.

S-PT84 suppresses vascular hyperpermeability by tail vein injection of Evans blue

Mucosal hypertrophy was observed in the segments around the patchy whitish plaques (Fig. 4), indicating damage resulting from Candidainduced edema. Therefore, we assessed the enhancement of vascular permeability by tail vein injection of Evans blue. Following intravenous administration of the dye, the gastric corpus was stained dark blue in the control (Candidainfected) group (Fig. 5B), in contrast to the limited staining observed in the normal (no Candida infection) group (Fig. 5A). Notably, Evans blue accumulation was also decreased in Candidainfected animals treated with S-PT84 (Fig. 5C). The amount of pigment was significantly increased in the control group compared with the normal group ( $0.337 \pm 0.095$ vs. $0.097 \pm 0.045$, respectively; $p=0.004)$; while pigment accumulation was tendency to decrease in the S-PT84 group compared to the control group $(0.232 \pm 0.028$ vs. 0.337 \pm 0.095 , respectively; $p=0.079$ ) (Fig. 6A). The incorporation of Evans blue was positively correlated with the stomach lesion score $(r=0.815, p=$ 0.014 ) (Fig. 6B). The regression line calculated using the incorporation of Evans blue as outcome variable ( $y$ ) and score of lesion as predictor variable $(x)$ was $y=0.0671 x+0.0662$. 


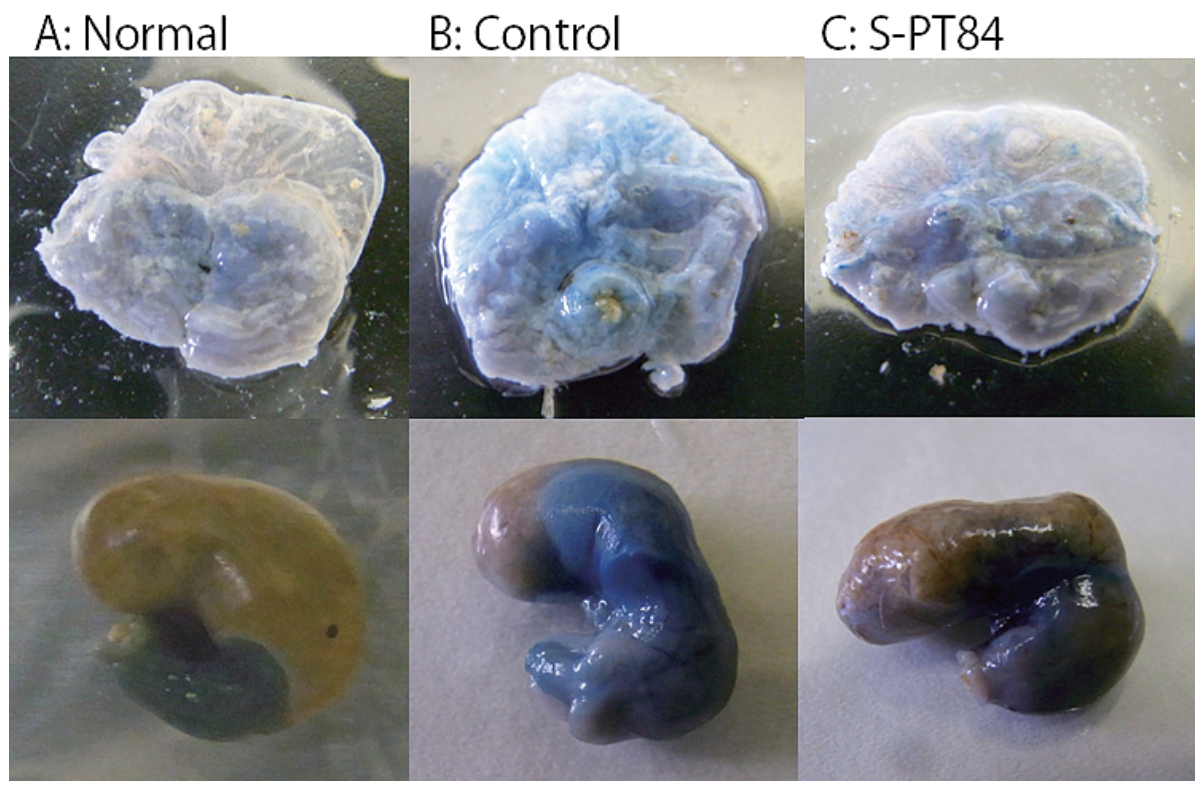

Fig. 5 Typical images of vascular permeability assessment by tail vein injection of Evans blue.

(A) Normal group (non-infected), (B) Control group (infected), (C) S-PT84 group (infected + S-PT84). Stomachs were recovered 20 minutes after tail vein injection of Evans blue. The stomachs were cut open along the longitudinal axis.

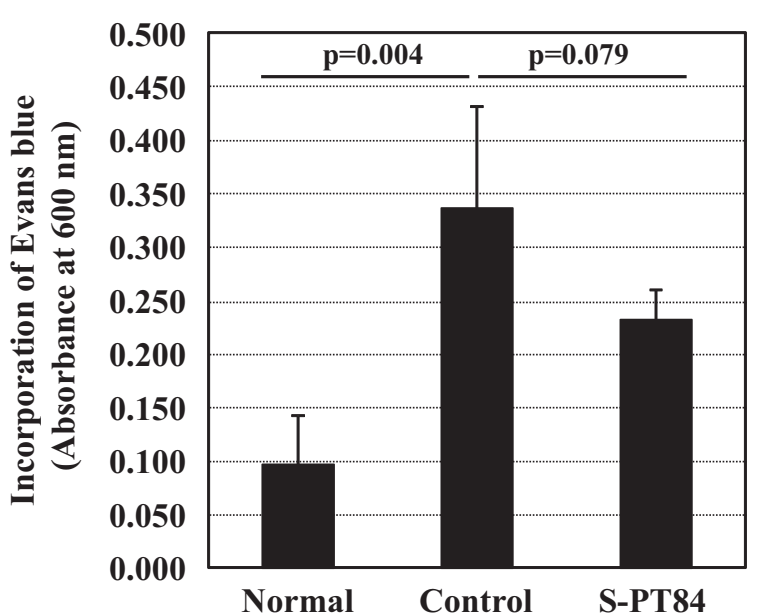

(A)

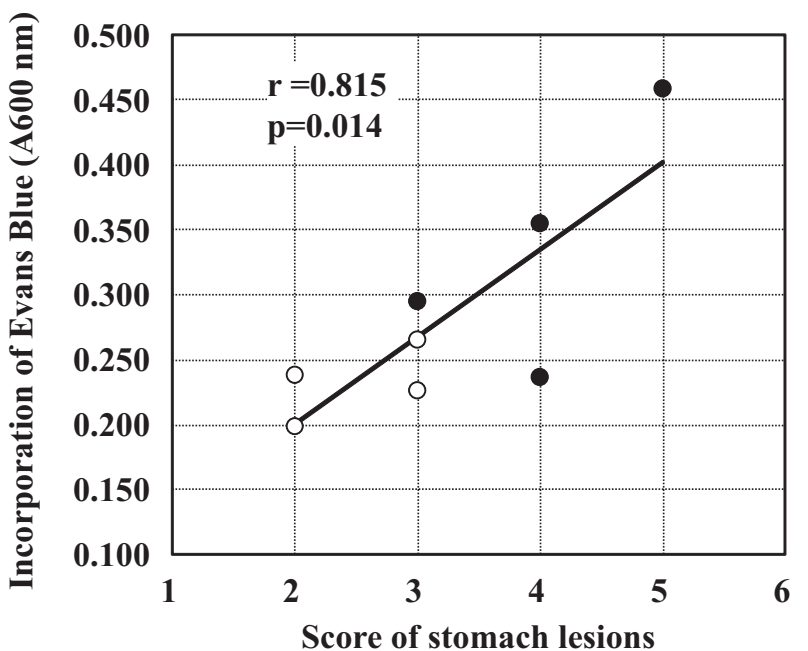

(B)

Fig. 6 Accumulation of Evans blue in the stomach following tail vein injection and the relationship between the incorporation of evans blue and score of stomach lesion.

Evans blue injection was performed as described in Fig. 5 legend. (A) Pigment accumulation was assessed as described in Methods. The optical absorbance at $600 \mathrm{~nm}$ was detected. Data are presented as mean $+S D(n=4)$. Significant differences were determined using unpaired Student's t-test. (B) The correlation was determined by Pearson's correlation coefficient test. Closed circles are control group (infected), open circles are S-PT84 group (infected + S-PT84).

S-PT84 prevents the adhesion of C. albicans and suppresses gastric inflammation in a mouse model of gastrointestinal candidiasis

Fig. 7 shows representative histopathological features of stomach mucosa in a mouse model of gastrointestinal candidiasis. In specimens from the normal group, the mucosal epithelia did not contain any $C$. albicans cells (Fig. 7A1 and 7A2). In contrast, a large amount of $C$. albicans (indicated by dark red staining) was detected on the mucosal epithelia from control group animals (Fig. 7B1 and 7B2), with C. albicans extending hyphae 


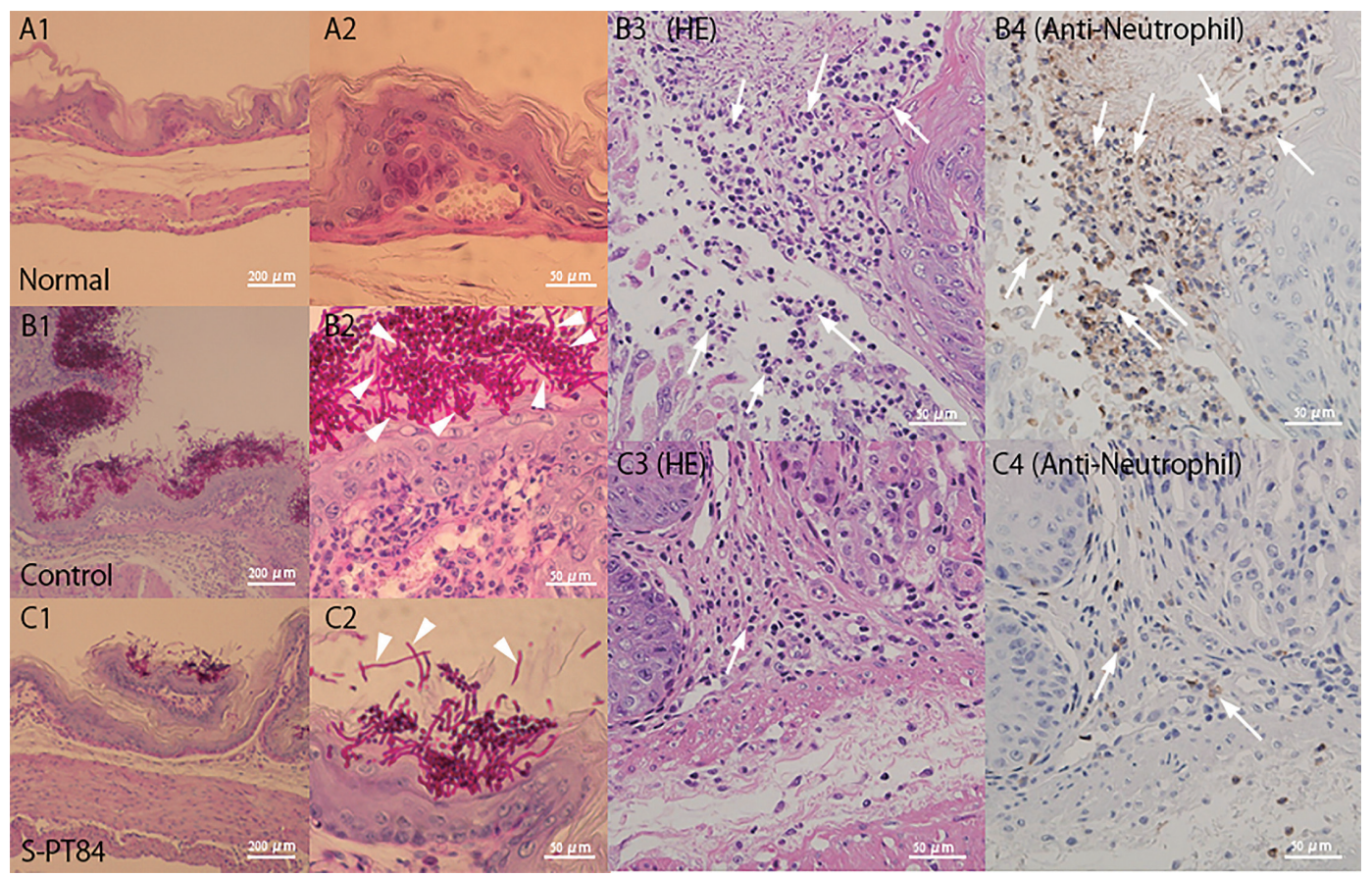

Fig. 7 Histology of cross-section of formalin-fixed-paraffin-embedded (FFPE) tissues of stomachs infected with $C$. albicans strain TIMM1768.

Mice were allocated to three groups: (A) Normal group (non-infected), (B) Control group (infected), (C) S-PT84 group (infected + S-PT84). To detect fungal cells, sections were stained using the Periodic Acid-Schiff (PAS) reaction and counter-stained with hematoxylineosin (HE). To detect neutrophil infiltration, sections were stained with anti-neutrophil antibody and counter-stained with hematoxylin. Arrowheads indicate PAS-stained C. albicans of mycelial form. Arrows indicate infiltrating neutrophils.

from the mucus into the lamina propria (indicated by arrowheads). Epithelium from the control group exhibited infiltration of polymorphonuclear cells into the submucosa (arrows, Fig. 7B3; enlarged view of the lower half part of Fig. 7B2), and these cells were identified as neutrophils by anti-neutrophil antibody staining (Fig. 7B4; about the same position of Fig. 7B3). In contrast, the density of Candida cells was visibly decreased by oral administration of S-PT84 (Fig. 7C1 and 7C2), as was the number of neutrophils infiltrating to the submucosa (Fig. 7C3 and 7C4; Fig. 7C3 is an enlarged view of the lower half part of Fig. 7C2, and Fig. 7C4 is about the same position of Fig. 7C3).

\section{Discussion}

In this study, S-PT84 was observed to adhere to the hyphal and yeast forms of $C$. albicans, and S-PT84 strongly inhibited the mycelial growth of $C$. albicans cells against the hyphal form selectively in vitro. In addition, the severity of stomach lesions and visible level of inflammation were decreased by in vivo administration of S-PT84.

S-PT84 also selectively suppressed metabolic activity of the hyphal form of $C$. albicans, leading to death of this cell type. We speculate that some component of S-PT84 with antifungal activity against the hyphal form of $C$. albicans might be involved in suppressing the effects of Candida infection. Nyanzi et al. reported that methanol extracts (containing 6-O-( $a_{-\mathrm{D}}-$ glucopyranosyl)-1 and 6-di-O-pentadecanoyl- $\alpha$-o -glucopyranose) from freeze-dried probiotics exhibited antiCandida activity ${ }^{22)}$; Li et al. reported that exopolysaccharide (EPS) from Lactobacillus plantarum R315 (which is genetically closely related to Lactobacillus pentosus) has antimicrobial activity against several pathogens, including $C$. albicans ${ }^{23)}$. These reports suggested that some components (such as 6-O-( $a$-o-glucopyranosyl)-1, 6-di-O-pentadecanoyl- $\alpha$ - - -glucopyranose, or EPS) can adhere to $C$. albicans, and that these interactions may mediate bacterial inhibition of $C$. albicans growth and/or virulence. In previous studies (data not shown), we have demonstrated (using the Burri method) that S-PT84 produces 
EPS; we hypothesize that this EPS might affect $C$. albicans growth. Further work is required to clarify which components of S-PT84 inhibit mycelial growth and could be useful in preventing Candida infection.

S-PT84 showed greater efficacy in decreasing the stomach lesion induced by $C$. albicans infection than did L. gasseri JCM $1131^{\top}$. In this study, the dosages of S-PT84 and L. gasseri JCM $1131^{\top}$ by oral administration were $4.5 \times 10^{9}$ cells/mouse and 9.18 $\times 10^{9}$ cells/mouse, respectively, and S-PT84 showed greater efficacy at lower dose than $L$. gasseri JCM $1131^{\top}$. Therefore, this result was consistent with the in vitro portion of the present study. On the other hand, the density of viable Candida cells in the stomachs of infected mice was not significantly decreased (compared to the control group) following bacterial treatment. In this context, we note that farnesol, a quorumsensing molecule for $C$. albicans, inhibits mycelial growth of $C$. albicans on the tongue surface and improves severity scores of tongues in murine oral candidiasis in a dose-dependent manner ${ }^{24)}$. However, in that previous experiment, the viable cell count of $C$. albicans was not decreased on the tongue (nor in feces, kidney, or liver) by treatment with farnesol. Notably, the CFU quantification method favors the detection of the yeast form of $C$. albicans, and farnesol appears to inhibit the change from yeast to hyphal cell type. Additionally, the same phenomenon (failure to detect decreased Candida cell density) was observed in a previous report ${ }^{20)}$. S-PT84 does not appear to act on the yeast form of $C$. albicans, whereas it apparently inhibits or kills the hyphal form of $C$. albicans by binding to the hyphal cell type (Fig. 2). This phenomenon may explain why the viable cell number was not significantly decreased, consistent with the lack of effect on cell number observed with farnesol treatment. On the other hand, the number of $C$. albicans cells stained with PAS was visibly decreased following treatment with S-PT84 (compare Fig. 7B1 with 7C1), suggesting that the total number of viable $C$. albicans cells in the S-PT84 group could be less than that in the control group. Hayama et al. reported that the administration of S-PT84 three times in the cavity and in the stomach decreased the number of $C$. albicans cells in the stomach in a murine oral candidiasis model. In regard to that report, we used a murine gastrointestinal model directly infected with $C$. albicans in the stomach and administered S-PT84 in the stomach only once. This suggests that the frequency of administration and the difference of the murine model might also affect the number of viable $C$. albicans cells in the stomach. Further studies are needed to confirm whether S-PT84 treatment indeed provides protection from candidiasis without actually altering the density of viable Candida cells in the stomach.

In the present work, we confirmed that $C$. albicans hyphae extended from the mucus to the lamina propria (Fig. 7B1 and 7B2), thereby permitting the infiltration of neutrophils into the submucosa (Fig. 7B3 and 7B4). In contrast, S-PT84 prevented the adhesion of $C$. albicans (Fig. $7 \mathrm{C} 1$ and $7 \mathrm{C2}$ ) and suppressed the infiltration of neutrophils into the submucosa following Candida infection (Fig. 7C3 and 7C4). From these results, we thought that suppression of the infiltration of neutrophils could be the result of the prevention of the adhesion of $C$. albicans. Additionally, SPT84 treatment provided an attenuation of the permeability of the stomach (as assayed using Evans blue accumulation) associated with Candida infection, and the extent of dyeing tended to decrease (Fig. 6A; $p=0.079$ ). Previously, we reported that ingestion of S-PT84 provides antiallergic effects by modulating the $\mathrm{T}$ helper ( $\mathrm{Th}$ ) type 1/type 2 balance toward a Th1-dominant state and by inducing regulatory $T$ cells ${ }^{16)}$. That study suggested that S-PT84 has the potential to modulate systemic inflammation via immune modulation; we therefore postulated that these effects could also contribute to the prevention of gastrointestinal inflammation. Indeed, Ninomiya et al. reported that neutrophils accumulated at the sites of Candida infection, and that terpinen-4-ol (a main constituent of tea tree oil) significantly suppressed the infiltration of neutrophils on the tongue's surface, ameliorating the severity of Candida infected lesions on this organ ${ }^{25,26)}$. We speculated that S-PT84's ability to modulate systemic inflammation could also result in decreased infiltration of neutrophils into infected stomach tissue, decreasing the severity scores of stomach lesions.

The induction of inflammation by Candida colonization may also occur in the intestinal tract $^{7)}$. Sonoyama et al. reported that $C$. albicans gut colonization increased the infiltration of inflammatory cells into the gut and induced gut hyperpermeability ${ }^{27)}$. This "leaky gut" symptomol- 
ogy is associated with several diseases, such as rheumatoid arthritis, diabetes, cirrhosis, nonalcoholic fatty liver disease (NAFLD), and irritable bowel syndrome (IBS) ${ }^{27-32)}$. These references suggest that the colonization of the digestive tract by $C$. albicans could be a cause of gastrointestinal inflammation, thereby inducing "leaky gut" and aggravating a variety of diseases.

In this study, we reported that oral administration of $L$. pentosus strain S-PT84 protected against C. albicans infection and ameliorated gastric inflammation in a murine model of gastrointestinal candidiasis. S-PT84 could be a useful tool for the prophylaxis and treatment of candidiasis in the intestinal tract and related syndromes associated with a leaky gut phenotype. Further experiments are needed to clarify the efficacy and mechanism of this probiotic treatment for candidiasis of the intestinal tract.

\section{Conflict of Interest}

T. Maekawa, M. Ida, T. Izumo, Y. Ono, and H. Shibata are employees of Suntory Wellness, Ltd., a company that markets health food products, including S-PT84. Research funding was provided to $S$. Abe and S. A. Ishijima by Suntory Wellness Ltd. The authors report no other conflicts of interest regarding this study.

\section{Author Contributions}

T. Maekawa participated in experimental design, analysis of the data, and drafting of the manuscript. M. Ida, T. Izumo, Y. Ono, and $\mathrm{H}$. Shibata participated in experimental design. S. A. Ishijima and $\mathrm{S}$. Abe participated in planning and conduct of the experiment, analysis of the data, and interpretation of the findings.

\section{References}

1) Miranda LN, van der Heijden IM, Costa SF, Sousa AP, Sienra RA, Gobara S, Santos CR, Lobo RD, Pessoa VP Jr, Levin AS: Candida colonisation as a source for candidaemia. J Hosp Infect 72: 9-16, 2009.

2) Oksuz S, Sahin I, Yildirim M, Gulcan A, Yavuz T, Kaya D, Koc AN: Phospholipase and proteinase activities in different Candida species isolated from anatomically distinct sites of healthy adults. Jpn J Infect Dis 60: 280-283, 2007.

3) Tsang CS, Chu FC, Leung WK, Jin LJ, Samar- anayake LP, Siu SC: Phospholipase, proteinase and haemolytic activities of Candida albicans isolated from oral cavities of patients with type 2 diabetes mellitus. J Med Microbiol 56: 1393-1398, 2007.

4) Katoh T, Maruyama R, Nishioka K: Isolation of Candida albicans from tongues showed no fungus by direct examination. Nippon Ishinkin Gakkai Zasshi 36: 145-148, 1995.

5) Vecchiarelli A, Pericolini E, Gabrielli E, Pietrella D: New approaches in the development of a vaccine for mucosal candidiasis: progress and challenges. Front Microbiol 3: 294, 2012.

6) Sasaki K: Candida-associated gastric ulcer relapsing in a different position with a different appearance. World J Gastroenterol 18: 4450-4453, 2012.

7) Kumamoto CA: Inflammation and gastrointestinal Candida colonization. Curr Opin Microbiol 14: 386-391, 2011.

8) Massarrat S, Saniee P, Siavoshi F, Mokhtari R, Mansour-Ghanaei F, Khalili-Samani S: The Effect of Helicobacter pylori infection, aging, and consumption of proton pump inhibitor on fungal colonization in the stomach of dyspeptic patients. Front Microbiol 7: 801, 2016.

9) Peleg AY, Hogan DA, Mylonakis E: Medically important bacterial-fungal interactions. Nat Rev Microbiol 8: 340-349, 2010.

10) Shirtliff ME, Peters BM, Jabra-Rizk MA: Crosskingdom interactions: Candida albicans and bacteria. FEMS Microbiol Lett 299: 1-8, 2009.

11) Holmes AR, McNab R, Jenkinson HF: Candida albicans binding to the oral bacterium Streptococcus gordonii involves multiple adhesin-receptor interactions. Infect Immun 64: 4680-4685, 1996.

12) Pereira-Cenci $T$, Deng DM, Kraneveld EA, Manders EM, Del Bel Cury AA, Ten Cate JM, Crielaard W: The effect of Streptococcus mutans and Candida glabrata on Candida albicans biofilms formed on different surfaces. Arch Oral Biol 53: 755-764, 2008.

13) Peters BM, Jabra-Rizk MA, Scheper MA, Leid JG, Costerton JW, Shirtliff ME: Microbial interactions and differential protein expression in Staphylococcus aureus-Candida albicans dual-species biofilms. FEMS Immunol Med Microbiol 59: 493-503, 2010.

14) Ogunshe AA, Omotoso MA, Bello VB: The in vitro antimicrobial activities of metabolites from lactobacillus strains on Candida species implicated in Candida vaginitis. Malays J Med Sci 18: 13-25, 2011.

15) Ishijima SA, Hayama K, Ninomiya K, Iwasa M, Yamazaki M, Abe S: Protection of mice from oral Candidiasis by heat-killed enterococcus faecalis, possibly through its direct binding to Candida albicans. Med Mycol J 55: E9-E19, 2014.

16) Nonaka $Y$, Izumo $T$, Izumi $F$, Maekawa $T$, Shibata $H$, Nakano A, Kishi A, Akatani K, Kiso Y: Antiallergic effects of Lactobacillus pentosus strain S-PT84 
mediated by modulation of Th1/Th2 immunobalance and induction of IL-10 production. Int Arch Allergy Immunol 145: 249-257, 2008.

17) Izumo T, Maekawa T, Ida M, Noguchi A, Kitagawa $\mathrm{Y}$, Shibata $\mathrm{H}$, Yasui $\mathrm{H}$, Kiso Y: Effect of intranasal administration of Lactobacillus pentosus S-PT84 on influenza virus infection in mice. Int Immunopharmacol 10: 1101-1106, 2010.

18) Izumo T, Izumi F, Nakagawa I, Kitagawa $Y$, Shibata $\mathrm{H}$, Kiso Y: Influence of Lactobacillus pentosus SPT84 ingestion on the mucosal immunity of healthy and Salmonella typhimurium-infected mice. Biosci Microflora 30: 27-35, 2011.

19) Hayama K, Ishijima $S$, Ono $Y$, Izumo $T$, Ida $M$, Shibata H, Abe S: Protective activity of S-PT84, a heat-killed preparation of Lactobacillus pentosus, against oral and gastric candidiasis in an experimental murine model. Med Mycol J 55: J123-129, 2014.

20) Ishijima SA, Abe S: A Novel Murine Candidiasis Model with severe colonization in the stomach induced by $\mathrm{N}$-acetylglucosamine-treatment and its scoring system based on local characteristic stomach symptoms. Med Mycol J 56: E31-E39, 2015.

21) Ishijima SA, Hayama K, Burton JP, Reid G, Okada M, Matsushita Y, Abe S: Effect of Streptococcus salivarius $\mathrm{K} 12$ on the in vitro growth of Candida albicans and its protective effect in an oral candidiasis model. Appl Environ Microbiol 78: 2190-2199, 2012.

22) Nyanzi R, Awouafack MD, Steenkamp $P$, Jooste PJ, Eloff JN: Anticandidal activity of cell extracts from 13 probiotic Lactobacillus strains and characterisation of lactic acid and a novel fatty acid derivative from one strain. Food Chem 164: 470-475, 2014.

23) Li S, Huang R, Shah NP, Tao X, Xiong Y, Wei H: Antioxidant and antibacterial activities of exopolysaccharides from Bifidobacterium bifidum WBIN03 and Lactobacillus plantarum R315. J Dairy Sci 97: 7334-7343, 2014.

24) Hisajima $T$, Maruyama $N$, Tanabe $Y$, Ishibashi $H$, Yamada T, Makimura K, Nishiyama Y, Funakoshi $\mathrm{K}$, Oshima H, Abe S: Protective effects of farnesol against oral candidiasis in mice. Microbiol Immunol 52: 327-333, 2008.
25) Ninomiya K, Hayama K, Ishijima SA, Maruyama N, Irie H, Kurihara J, Abe S: Suppression of inflammatory reactions by terpinen-4-ol, a main constituent of tea tree oil, in a murine model of oral candidiasis and its suppressive activity to cytokine production of macrophages in vitro. Biol Pharm Bull 36: 838-844, 2013.

26) Ninomiya $K$, Maruyama $N$, Inoue $S$, Ishibashi $H$, Takizawa T, Oshima H, Abe S: The essential oil of Melaleuca alternifolia (tea tree oil) and its main component, terpinen-4-ol protect mice from experimental oral candidiasis. Biol Pharm Bull 35: 861-865, 2012.

27) Sonoyama K, Miki A, Sugita R, Goto $H$, Nakata M, Yamaguchi N: Gut colonization by Candida albicans aggravates inflammation in the gut and extra-gut tissues in mice. Med Mycol 49: 237-247, 2011.

28) Sapone A, de Magistris L, Pietzak M, et al: Zonulin upregulation is associated with increased gut permeability in subjects with type 1 diabetes and their relatives. Diabetes 55: 1443-1449, 2006.

29) Lassenius MI, Pietiläinen KH, Kaartinen K, Pussinen PJ, Syrjänen J, Forsblom C, Pörsti I, Rissanen A, Kaprio J, Mustonen J, Groop PH, Lehto M; FinnDiane Study Group: Bacterial endotoxin activity in human serum is associated with dyslipidemia, insulin resistance, obesity, and chronic inflammation. Diabetes Care 34: 1809-1815, 2011.

30) Goyal A, Ghoshal UC, Ahamad I, Roy R, Srivastava D, Mohindra S, Saraswat VA, Khetrapal CL: Frequency and factors associated with increased small intestinal permeability in patients with portal hypertension. Trop Gastroenterol 34: 136-143, 2013.

31) Miele L, Valenza V, La Torre G, Montalto M, Cammarota G, Ricci R, Mascianà R, Forgione A, Gabrieli ML, Perotti G, Vecchio FM, Rapaccini G, Gasbarrini G, Day CP, Grieco A: Increased intestinal permeability and tight junction alterations in nonalcoholic fatty liver disease. Hepatology 49 : 1877-1887, 2009.

32) Shulman RJ, Eakin MN, Czyzewski DI, Jarrett M, Ou CN: Increased gastrointestinal permeability and gut inflammation in children with functional abdominal pain and irritable bowel syndrome. J Pediatr 153: 646-650, 2008. 